\title{
Correlation between antifungal consumption and the distribution of Candida species in different hospital departments of a Lebanese medical Centre
}

Lyn Awad ${ }^{1 \dagger}$, Hani Tamim²+, Dania Abdallah ${ }^{3}$, Mohammad Salameh ${ }^{4}$, Anas Mugharbil ${ }^{5}$, Tamima Jisr ${ }^{6}$, Kamal Zahran ${ }^{7}$, Nabila Droubi ${ }^{8}$, Ahmad Ibrahim ${ }^{5}$ and Rima Moghnieh ${ }^{9^{*}}$

\begin{abstract}
Background: In recent years, there has been a significant increase in the incidence of fungal infections attributed to Candida species worldwide, with a major shift toward non-albicans Candida (NAC). In this study, we have described the distribution of Candida species among different hospital departments and calculated the antifungal consumption in our facility. We also correlated the consumption of certain antifungals and the prevalence of specific Candida species.

Methods: This was a retrospective review of all the Candida isolates recovered from the computerised microbiology laboratory database of Makassed General Hospital, a tertiary care centre in Beirut, Lebanon, between January 2010 and December 2015. Data on antifungal consumption between January 2008 and December 2015 were extracted from the hospital pharmacy electronic database. We used Spearman's coefficient to find a correlation between Candida species distribution and antifungal consumption.

Results: Between 2008 and 2015, we observed that the highest antifungal consumption was in the haematology/ oncology department (days of therapy/1000 patient days $=348.12 \pm 85.41$ ), and the lowest was in the obstetrics/ gynaecology department $(1.36 \pm 0.47)$. In general, the difference in antifungal consumption among various departments was statistically significant $(P<0.0001)$. Overall, azoles were the most common first-line antifungals in our hospital. Echinocandins and amphotericin B were mostly prescribed in the haematology/oncology department. As for Candida species distribution, a total of 1377 non-duplicate isolates were identified between 2010 and 2015. A non-homologous distribution of albicans vs. non-albicans was noted among the different departments $(P=0.02)$. The most commonly isolated NAC was Candida glabrata, representing 14\% of total Candida species and 59\% of NAC. Candida famata ( $9 \%$ of NAC), Candida parapsilosis (3.6\% of NAC) and Candida krusei (3\% of NAC) were recovered unequally from the different departments. The total antifungal consumption correlated positively with the emergence of NAC. The use of azoles correlated positively with Candida glabrata, while amphotericin B formulations correlated negatively with it. None of these correlations reached statistical significance.
\end{abstract}

Conclusion: Different Candida species were unequally distributed among different hospital departments, and this correlated with consumption of antifungals in respective departments, highlighting the need for antifungal stewardship.

Keywords: Amphotericin B, Antifungal, Azoles, Candida albicans, Candida glabrata, Candida famata, Consumption, Correlation, Critical care, Echinocandins, Non-albicans Candida, Obstetrics, Oncology

\footnotetext{
* Correspondence: moghniehrima@gmail.com

${ }^{+}$Lyn Awad and Hani Tamim contributed equally to this work.

${ }^{9}$ Head of Antimicrobial Stewardship Program, Makassed General Hospital,

Beirut, Lebanon

Full list of author information is available at the end of the article
}

(c) The Author(s). 2018 Open Access This article is distributed under the terms of the Creative Commons Attribution 4.0 International License (http://creativecommons.org/licenses/by/4.0/), which permits unrestricted use, distribution, and reproduction in any medium, provided you give appropriate credit to the original author(s) and the source, provide a link to the Creative Commons license, and indicate if changes were made. The Creative Commons Public Domain Dedication waiver (http://creativecommons.org/publicdomain/zero/1.0/) applies to the data made available in this article, unless otherwise stated. 


\section{Background}

In recent years, the world has witnessed a significant increase in the incidence of fungal infections due to Candida species [1], with Candida albicans (CA) being the most common causative organism [2]. However, recent studies have documented a change in this aetiology shifting toward non-albicans Candida (NAC) [3]. This shift has been linked to the selective pressure caused by the extensive use of broad spectrum antibiotics and antifungals [4]. For many years, azoles have been used as prophylactic agents against fungal infections in immunocompromised patients, as empiric/preemptive treatment of fungal disease in cancer or critically ill patients, in addition to their use as targeted therapy of Candida infections $[1,5]$. However, polyenes consumption, especially lipid formulations with amphotericin $\mathrm{B}$, has been used increasingly in the immunocompromised population [6], along with echinocandins, which were introduced into the market in 2002 [7].

The geographic distribution of Candida species may reflect the antimicrobial prescription habits in each healthcare facility $[8,9]$. Multiple studies have looked at the relative distribution of Candida species with time [3, 8 , 10]; however, few have studied its geographic distribution (i.e., in specific hospital departments along with corresponding antifungal expenditure) $[8,11]$. Two studies demonstrated an increase in the incidence of Candida parapsilosis associated with the use of caspofungin [12, 13].

In this study, our primary aim was to calculate the consumption of each class of antifungals, and to describe the relative distribution of different Candida species in different hospital departments. The secondary aim was to find a correlation between the expenditure of certain antifungals and the prevalence of specific Candida species.

\section{Methods}

\section{Setting and study design}

This was a retrospective review of all Candida isolates retrieved from the computerised microbiology laboratory database at Makassed General Hospital (MGH), between January 2010 and December 2015. The MGH Institutional Review Board Committee granted this study approval. No informed consent was required due to it retrospective nature. The reporting of this study conforms to the STROBE statement [14]. MGH is a 186-bed university hospital in Beirut, Lebanon. The monthly occupancy rate ranges between 70 and $80 \%$, with 17 beds in critical care, 71 beds in internal medicine (IM), 17 beds in haematology/oncology and bone marrow transplantation, 21 beds in surgery, 13 beds in obstetrics/gynaecology (OBGYN) and 47 beds in the paediatric departments. All Candida species reported in the database were selected, and non-duplicate isolates recovered during the study period were included in this study. All specimens from different culture sites, such as abscess, bronchoalveolar lavage, blood, catheter, ear, eye, fluid, sputum, deep-tracheal aspirate, throat, urine, vagina and wound were included, except for stool specimens.

\section{Identification and speciation of Candida isolates}

The identification and speciation of Candida isolates were performed according to the microscopic and macroscopic growth morphology and germ tube test. Isolates producing germ tubes within $3 \mathrm{~h}$ of incubation were further differentiated. Speciation of the Candida isolates was performed using the API 20 C AUX system (bio Merieux, France) [15]. Results were interpreted after 48 to $72 \mathrm{~h}$ of incubation at $29^{\circ} \mathrm{C} \pm 2{ }^{\circ} \mathrm{C}$. Antifungal susceptibility testing is not available in our institution.

\section{Protocol for antifungal use in our hospital}

Broad-spectrum antifungals were prescribed on hospital setting for the management of invasive fungal infections according to institutional guidelines on antimicrobial use, which were based on international guidelines [16, 17]. Patient colonisation with Candida spp. such as in urine, sputum, skin or others was not usually treated with antifungals. Patients with evidence of candidemia or other invasive Candida infections were treated with antifungals [16]. Neutropenic patients with cancer were given prophylactic, empiric, pre-emptive or targeted antifungal therapy as per the clinical need [17]. In ICU, newly diagnosed septic patients without an evident focus of infection were evaluated by an Infectious Disease physician for the possibility of adding on systemic antifungals to their treatment regimens in the following cases: having already received broad-spectrum antibacterial therapy active against our nosocomial flora and having been colonized with Candida species. Systemic infections due to C. albicans and C. tropicalis were managed using fluconazole, while other NAC infections except for those caused by C. krusei were treated using echinocandins $[16,18]$. C. krusei-related infections were given lipid formulation amphotericin $\mathrm{B}[16,18]$.

\section{Definitions \\ Non-duplicate isolates}

When multiple isolates were obtained from the same patient, all species were included in the study, but only the first isolate of a given species was considered in the analysis [8].

\section{Days of therapy (DOT)}

The number of days that a patient was on an antimicrobial regardless of the dose [19]. 


\section{Defined daily dose (DDD)}

Corresponds to the assumed average daily dose of an antimicrobial for its main indication in adults based on the World Health Organisation Anatomical Therapeutic Chemical (WHO/ATC) classification system for each antifungal [20].

\section{Patient days (PD)}

Calculated by counting the number of patients present in any given location (e.g., hospital or ward) at a single time during a 24-h period [21].

\section{Antifungal consumption}

Data on antifungal consumption between January 2008 and December 2015 were extracted from the electronic database of the hospital pharmacy. Antifungals were categorised according to their pharmacological class: azoles (fluconazole and voriconazole), echinocandins (caspofungin, anidulafungin, micafungin) and polyenes (conventional amphotericin B and lipid formulations amphotericin B). We used two types of metrics to measure antifungal expenditure: DDD/1000 PD, and DOT/1000 PD [20, 22].

In paediatrics, the use of DOT is preferred because the antimicrobial doses are adjusted according to body weight, and there is no universal DDD [22]. In order to compare the antifungal consumption in the different hospital departments, including the paediatric department DOT/ $1000 \mathrm{PD}$ had to be used. In the critical care and haematology/oncology departments, we used DDD/1000 PD to compare and benchmark with other studies in the published literature.

The DDD was $200 \mathrm{mg}$ for fluconazole, $400 \mathrm{mg}$ for voriconazole, $100 \mathrm{mg}$ for anidulafungin, $50 \mathrm{mg}$ for caspofungin and $100 \mathrm{mg}$ for micafungin [20]. 19 There is no standardised DDD for the lipid formulations of polyenes [22, 23]. There is only a DDD for the amphotericin B deoxycholate (Fungizone $^{\circ}$ ), which is $35 \mathrm{mg}$. Thus, for lipid-based formulations, we defined the DDD based on the regular daily dose used in our facility, which is $300 \mathrm{mg}$ for both the liposomal and the lipid complex formulations.

\section{Statistical analysis}

The Statistical Package for Social Sciences (SPSS, version 21) program was used for data entry, management and analysis. Categorical variables are presented as number and percent, whereas continuous variables are presented as mean and standard deviation. Bivariate analysis was carried out using the chi-squared test for comparing categorical variables, whereas continuous ones were compared using the Student's t-test. The relationship between antifungal usage and the distribution of NAC was determined using the Spearman's coefficient for non-parametric correlation. A $P<0.05$ was considered significant.

\section{Results}

\section{Antifungal consumption}

The rate of antifungal consumption in DOT/1000 PD and DDD/1000 PD are shown in Tables 1 and 2, respectively. All rates were reported as mean \pm standard deviation. From 2008 to 2015, the mean total antifungal consumption, in terms of DOT/1000 PD was $180.69 \pm 135.5$.

Results in the two metric methods (DOT/1000 PD and $\mathrm{DDD} / 1000 \mathrm{PD}$ ) revealed almost parallel patterns, with the exception of the azoles because the used daily doses of azoles in the hospital were much lower than the DDD, and were not consistent among different indications.

In general, the rate of antifungal consumption during the study period was not analogous among the different departments $(P<0.0001)$ (Table 1$)$. In terms of DOT/1000 $\mathrm{PD}$, total antifungal consumption was highest in the haematology/oncology department (348.12 \pm 85.41$)$, followed by critical care $(73.85 \pm 22.25)$, and was lowest in the OBGYN department $(1.36 \pm 0.47)$ (Table 1). The difference between the mean antifungal consumption in any two departments was statistically significant when compared to one another, with the exception of the consumption in the surgery department $(33.56 \pm 11.65)$ when compared to that in the paediatric department $(28.70 \pm 11.96)$, and the critical care department $(73.85 \pm 22.25)$ when compared to that in the IM department $(56.48 \pm 24.06)$, where the difference was non-significant $(P=0.565$ and 0.23 , respectively). Relative azole consumption mirrored total antifungal consumption, where the difference was statistically significant among the departments $(P<0.0001)$. Likewise, the highest consumption was seen in the haematology/oncology department (214.65 \pm 47.67$)$, and the lowest was seen in the OBGYN department $(0.94 \pm 1.10)$ (Table 1). Echinocandins

Table 1 Antifungal consumption in terms of DOT/1000 PD (mean \pm SD) among different hospital departments between 2008 and 2015

\begin{tabular}{|c|c|c|c|c|c|c|c|}
\hline \multirow{2}{*}{$\begin{array}{l}\text { Antifungal } \\
\text { Class }\end{array}$} & \multicolumn{7}{|c|}{ Hospital Department } \\
\hline & Critical care & Paediatric & Hem/Onc & OBGYN & Surgery & IM & $P$-value \\
\hline Azoles & $48.41 \pm 14.58$ & $23.29 \pm 9.24$ & $214.65 \pm 47.67$ & $0.94 \pm 1.10$ & $24.31 \pm 10.24$ & $46.44 \pm 9.50$ & $<0.0001$ \\
\hline Echinocandins & $21.13 \pm 27.26$ & $1.35 \pm 1.55$ & $67.96 \pm 19.04$ & $0.42 \pm 1.19$ & $7.20 \pm 11.25$ & $7.69 \pm 5.49$ & $<0.0001$ \\
\hline Amphotericin B & $4.32 \pm 3.62$ & $4.06 \pm 3.36$ & $65.50 \pm 18.98$ & 0 & $2.06 \pm 3.62$ & $2.34 \pm 1.49$ & $<0.0001$ \\
\hline Total & $73.85 \pm 22.25$ & $28.70 \pm 11.96$ & $348.12 \pm 85.41$ & $1.36 \pm 0.47$ & $33.56 \pm 11.65$ & $56.48 \pm 24.06$ & $<0.0001$ \\
\hline
\end{tabular}

KEY: Hem/Onc = Haematology/oncology, IM = Internal medicine, OBGYN= Obstetrics/gynaecology 
Table 2 Antifungal consumption in terms of DDD/1000 PD (mean \pm SD) among different hospital departments between 2008 and 2015

\begin{tabular}{llllll}
\hline Antifungal & \multicolumn{3}{l}{ Hospital Department } & & \\
\cline { 2 - 6 } & Critical care & Hem/Onc & OBGYN & Surgery & IM \\
\hline Azoles & $33.79( \pm 10.98)$ & $146.51( \pm 41.28)$ & $0.56( \pm 1.03)$ & $22.76( \pm 9.97)$ & $39.73( \pm 7.25)$ \\
Echinocandins & $11.36( \pm 8.33)$ & $57.96( \pm 25.48)$ & 0 & $4.38( \pm 8.27)$ & $7.26( \pm 6.92)$ \\
Amphotericin B & $4.29( \pm 4.36)$ & $79.83( \pm 30.56)$ & 0 & $0.50( \pm 0.98)$ & $1.93( \pm 1.60)$ \\
\hline
\end{tabular}

KEY: Hem/Onc = Haematology/oncology, IM = Internal medicine, OBGYN = Obstetrics/gynaecology

were mostly used in oncology $(67.96 \pm 19.04)$, followed by a significantly lower consumption in critical care (21.13 $\pm 27.26, P=0.01)$, and almost a null consumption in the paediatric and OBGYN departments $(1.35 \pm 1.55$ and $0.42 \pm 1.19$ respectively, $P=0.2$ ) (Table 1 ). Finally, conventional or lipid formulations of amphotericin $\mathrm{B}$ were mostly used in the haematology/oncology department $(65.50 \pm 18.98)$, followed by a similar distribution in critical care and paediatric departments $(4.32 \pm 3.62$, and 4.06 \pm 3.36 , respectively, $P=0.885$ ), then to a lesser extent in the IM and surgery departments (mean $2.34 \pm 1.49$, and $2.06 \pm 3.62$, respectively, $P=0.840$ ). It was never used in the OBGYN department (Table 1). When antifungal consumption was measured in DDD/1000 PD, a similar trend was observed, excluding the paediatric department (Table 2).

\section{Candida isolates distribution}

Between 2010 and 2015, a total of 1377 non-duplicate Candida isolates were identified, including colonizers and pathogens. The majority of these isolates were recovered from urine (48\%), followed by the respiratory tract (20\% from deep-tracheal aspirate and 17\% from sputum), and only $2 \%$ were from blood (Fig. 1).

The highest number of isolates was collected from the IM department (49\%), followed by the critical care department (30\%), the surgery (6\%), paediatric $(6 \%)$, haematology/oncology (5\%) and OBGYN (4\%) departments (Fig. 2).

\section{General distribution of Candida species with a focus on NAC in different departments}

In all departments and NAC isolation was statistically significant among hospital departments $(P=0.02)$ (Table 3$)$.

In all departments, CA was the most commonly isolated species, representing $76 \%$ of total isolates (Fig. 3) and ranging from $91.4 \%$ in the OBGYN department to $70.8 \%$ in the surgery department (Table 4).

In terms of NAC, the OBGYN department had the lowest significant rate of NAC compared to the critical care, haematology/oncology and surgery departments $(P=0.01$, 0.02 , and 0.003 respectively).

The rate of NAC in the paediatric department was the second lowest compared to other hospital units (Table 4).

\section{Distribution of different species of NAC}

The most commonly isolated NAC in our facility was Candida glabrata (197 isolates), accounting for $14 \%$ of total isolates and $59 \%$ of total NAC. Non-speciated Candida followed, accounting for 3\% of total isolates and $13.5 \%$ of total NAC. Candida famata came in third place, representing $2 \%$ of total isolates and $9 \%$ of total

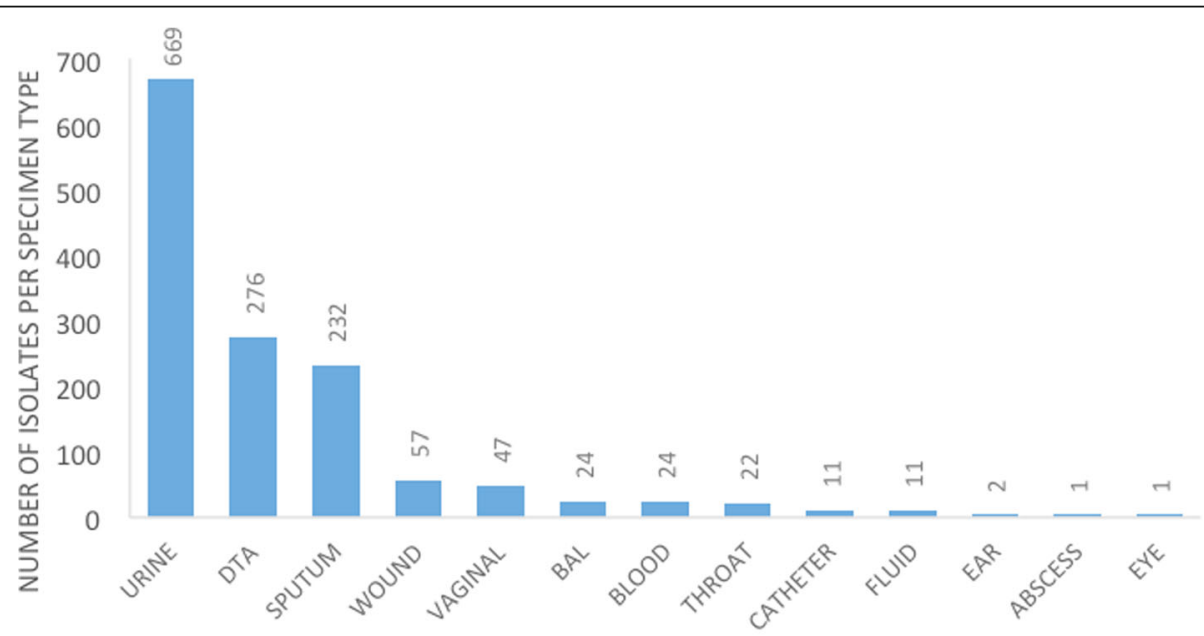

TYPE OF SPECIMEN

Fig. 1 Distribution of different specimens types growing Candida species. KEY: BAL = Bronchoalveolar lavage, DTA = Deep tracheal aspirate 


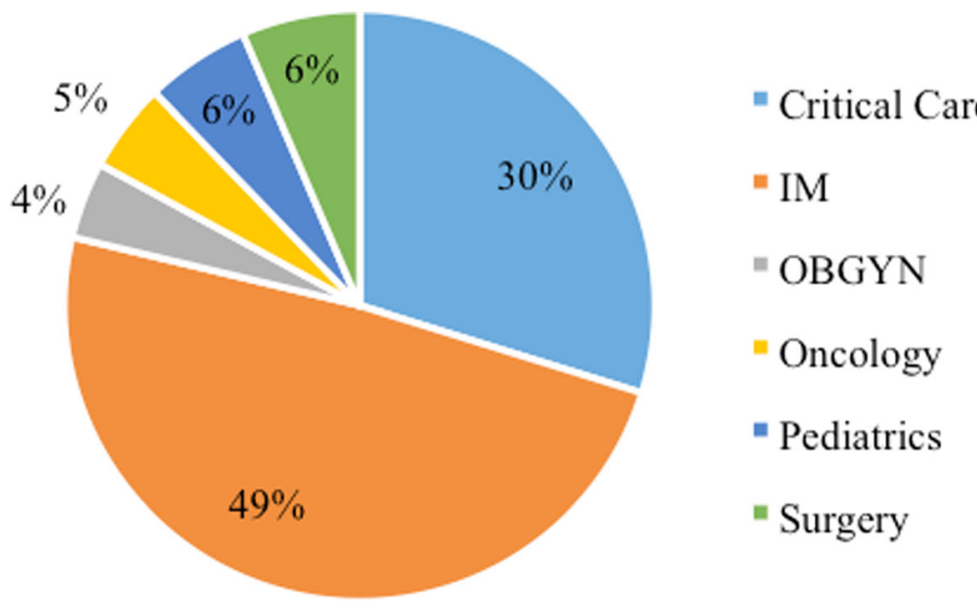

Fig. 2 Distribution of Candida isolates in the different hospital departments. KEY: IM = Internal medicine, OBGYN= Obstetrics/gynaecology

NAC. Candida krusei and Candida tropicalis represented 1 and $0.5 \%$ of the total isolates, respectively (Figs. 3 and 4 ).

\section{Distribution of Candida glabrata in different departments} The proportion of Candida glabrata among NAC differed among the units. In OBGYN, Candida glabrata has been the only isolated NAC (100\% of total NAC). The lowest rate of Candida glabrata among NAC was seen in the paediatric department (33\% of NAC), and the haematology/oncology departments (47\% of NAC) (Table 5) $(P=0.03)$.

\section{Distribution of NAC other than Candida glabrata}

The proportion of NAC other than Candida glabrata among different departments is presented in Table 3. There was less variability in the type of NAC in the OBGYN, haematology/oncology and paediatric departments in comparison with the IM, critical care and surgery departments.

The non-speciated Candida were mostly observed in the paediatric department (25\% of NAC), followed by

Table 3 Distribution of Candida albicans versus non-albicans Candida among hospital departments between 2010 and 2015 and comparison between them

\begin{tabular}{llll}
\hline Department & $\begin{array}{l}\text { Candida albicans } \\
(N=1044 \text { isolates })\end{array}$ & $\begin{array}{l}\text { Non-albicans Candida } \\
(N=333 \text { isolates })\end{array}$ & P-value \\
\hline Critical care & $313(76.3 \%)$ & $97(23.7 \%)$ & 0.02 \\
IM & $498(73.9 \%)$ & $176(26.1 \%)$ & \\
OBGYN & $53(91.4 \%)$ & $5(8.6 \%)$ & \\
Hem/Onc & $50(74.6 \%)$ & $17(25.4 \%)$ & \\
Paediatric & $67(84.8 \%)$ & $12(15.2 \%)$ & \\
Surgery & $63(70.8 \%)$ & $26(29.2 \%)$ & \\
\hline
\end{tabular}

KEY: Hem/Onc = Haematology/oncology, IM = Internal medicine, $O B G Y N=$ Obstetrics/gynaecology the surgery (15\% of NAC), IM (14\% of NAC), critical care $(12.4 \%$ of NAC) and oncology departments (6\% of NAC). They were absent in the OBGYN department.

Second to Candida glabrata among the speciated Candida, Candida famata ( $9 \%$ of NAC) was the most common in all departments, with the exceptions of the paediatric and OBGYN departments.

Candida krusei (3\% of NAC) was mostly recovered from the surgery and haematology/oncology departments, while it was absent from the OBGYN and paediatric departments.

Candida parapsilosis (3.6\% of NAC) had the highest percentage among the speciated Candida isolates second to Candida glabrata in the paediatric department (25\%).

\section{Correlation between antifungal consumption and the isolation of specific Candida species}

Using Spearman's coefficient, we observed that none of the correlations reached a statistical significance due to the limited number of hospital departments involved $(N=5)$; however, these results indicated some trends and showed clinical significance (Table 5).

The use of antifungals in general correlated positively with NAC [Spearman's Coefficient $(S C)=0.38$ ]. The use of amphotericin B showed a similar yet weaker positive correlation for the emergence of NAC in comparison with azoles and echinocandins ( $\mathrm{SC}=0.27$ vs. 0.40 and 0.39 , respectively). The effect of azoles and echinocandins on recovery of NAC was almost the same.

Regarding Candida glabrata alone, the use of azoles correlated positively with its emergence $(S C=0.13)$, unlike the use of amphotericin $\mathrm{B}$, which correlated negatively $(\mathrm{SC}=-0.003)$.

We noticed that in wards where amphotericin B had been used (haematology/oncology and paediatrics, 19 and $14 \%$ from total antifungal consumption in each ward, 


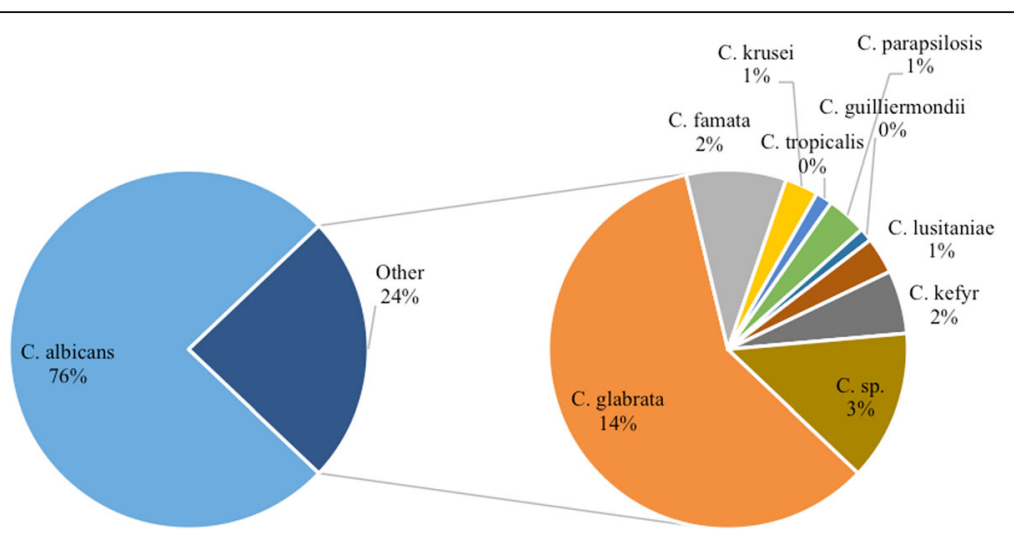

Fig. 3 Total distribution of the different Candida species in the hospital. KEY: C. sp. = Non-speciated Candida. N.B. Percentages are calculated from total Candida isolates recovered

respectively), there was less non-speciated Candida isolated from clinical specimens (Tables 1 and 5). We equally noticed less variability in the types of NAC recovered.

\section{Discussion}

The effect of antimicrobial use on the changing microbial ecology of inpatients in a specific healthcare facility is sure but slow, and the tangible consequences usually lag behind in time [24]. Accordingly, we reviewed antifungal consumption in different hospital departments from 2008 to 2015, and we studied the distribution of variable Candida species in the same departments during 2010 and 2015. Then, we attempted to find a correlation between the use of specific antifungals and the prevalence of specific Candida species.

\section{Antifungal consumption in different departments}

The discrepancies found between DOT and DDD per 1000 PD were due to the actual dosing of antifungals, especially the oral dosage form of fluconazole. The actual fluconazole doses that were used during the study period were below the DDD (200 $\mathrm{mg}$ actual dosing vs. $400 \mathrm{mg}$ recommended dosing) for indications, including oral thrush and vaginitis.

In Lebanon, like in the Middle East and North Africa region, there are limited data about antifungal consumption. In our study, azoles were the most commonly used antifungals. Similarly, Al Othman et al. studied the burden and treatment patterns of invasive fungal infections in Lebanon and in the Kingdom of Saudi Arabia, in which they found that fluconazole was the most commonly prescribed antifungal as a first-line therapy (69\%) [25]. The most common second-line antifungals were voriconazole (35\%)/caspofungin (30\%), followed by amphotericin B formulations in general [25]. In Europe, a multicentre French survey in 2012 involving 239 healthcare facilities similarly revealed that fluconazole was the most frequently used antifungal agent in haematology units and intensive care units (ICUs) [26]. Antifungal expenditure recorded its highest levels in participating cancer centres, followed by university hospitals [26]. In our hospital, the haematology/oncology department showed the highest antifungal consumption.

The comparison of our antimicrobial expenditure with other studies is hindered by the fact that the metrics are not standardised across hospitals and countries. Some use the recommended daily dose (RDD)/100 PD, such as Germany [27]. Others use DDD/1000 PD as in the formerly stated French study [26]. Some use DDD/1000 inhabitants, such as the European Centre for Disease Prevention and Control [28]. In paediatrics, because the calculations become even more complicated due to weight-based dosing, we used DOT/1000 PD [22].

\section{CA as the most commonly isolated species}

CA was the most commonly isolated species $(76 \%$ of total isolates) in our setting. So far in Lebanon, only two studies have described the distribution and epidemiology of variable Candida species in different medical institutions $[10,25]$. In both of them, CA was the most commonly isolated species throughout the years $(64 \%$ in 2007 [10], and 56\% in 2011 [25]). In another neighbouring country, Turkey, CA was found to make up 59.5\% of the total strains in various departments at the Izmir Hospital [29]. Similarly in Italy, CA was the most commonly isolated species $(72.7 \%)$ in different departments of a tertiary care hospital over a three-year period [2]. These results show that, although it is clear that the rate of recovery of NAC is increasing, CA remains the most common Candida species in general.

\section{Most commonly isolated NAC}

The predominant speciated NAC in our study was Candida glabrata (59\% of NAC, and 14\% of total Candida). This finding was different from other Lebanese studies, 


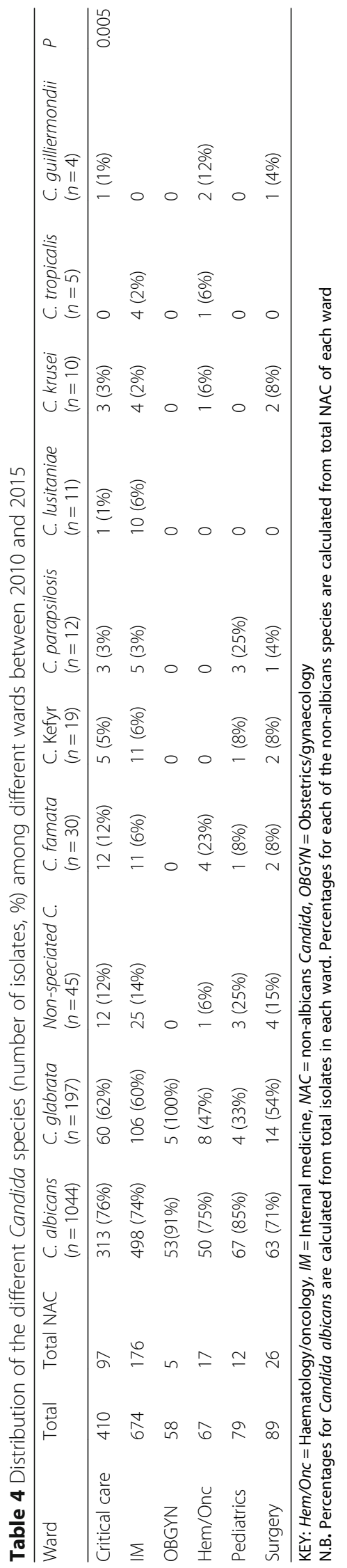




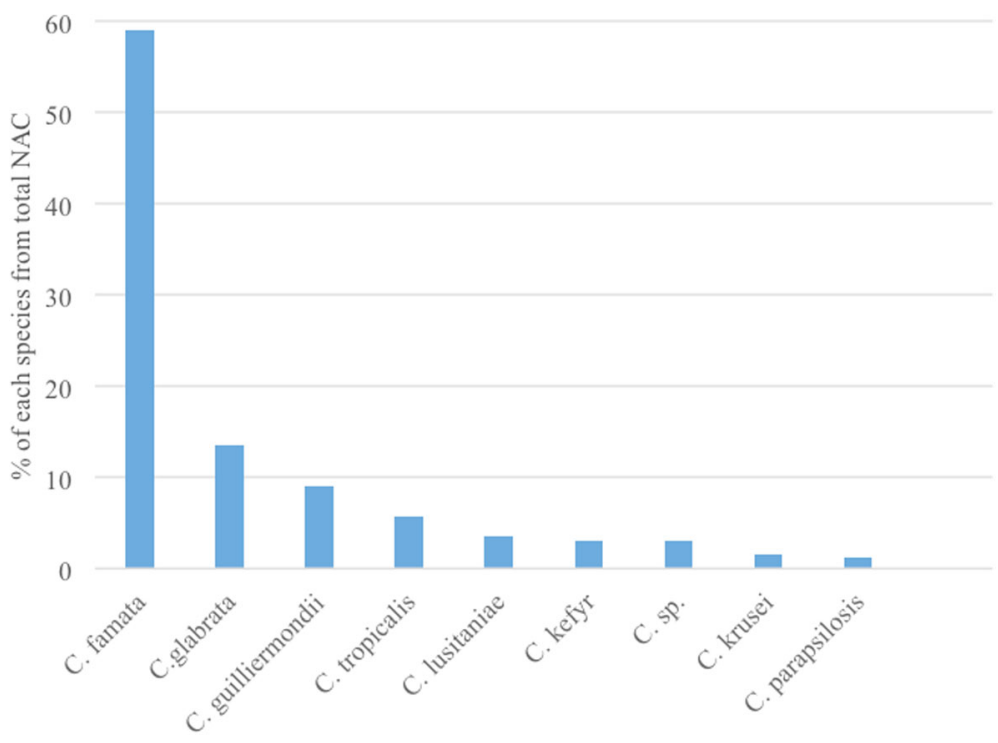

Fig. 4 Percentages of the different species of NAC from total NAC. KEY: C. sp. = Non-speciated Candida

in which Candida tropicalis was the most commonly isolated NAC (35-45\% [10], and 20\% [25], both of total isolates). This difference in species distribution between healthcare centres of the same country may be attributed to the selection bias used. In our study, we have included all non-faecal isolates of all departments, irrespective of their clinical significance, while in the two other studies $[10,25]$, the analysed isolates were retrieved from clinically relevant specimens, either when speciation was performed based on the treating clinicians' requests [10], or in patients who warranted the use of antifungal therapy in special clinical circumstances [25].

Similar to our findings, Candida glabrata predominated among NAC in many centres, such as in France (15\% of total) [8] and in Turkey (14.4\% of total) [29]. Yet, other NAC may prevail in other settings. For example, Candida krusei was the most common NAC

Table 5 Correlation of antifungal consumption (based on mean DOT/1000 PD) and non-albicans Candida isolation

\begin{tabular}{llll}
\hline & & $\begin{array}{l}\text { Non-albicans } \\
\text { Candida }\end{array}$ & $\begin{array}{l}\text { Candida } \\
\text { glabrata }\end{array}$ \\
\hline Total antifungals & Spearman's coefficient & 0.38 & 0.12 \\
& p-value & 0.46 & 0.83 \\
Azoles & Spearman's coefficient & 0.40 & 0.13 \\
& p-value & 0.43 & 0.80 \\
Echinocandins & Spearman's coefficient & 0.39 & 0.18 \\
& p-value & 0.43 & 0.73 \\
Amphotericin B & Spearman's coefficient & 0.27 & -0.003 \\
& p-value & 0.59 & 0.99 \\
\hline
\end{tabular}

N.B. A positive value (Spearman's coefficient) represents a positive correlation and a negative value (Spearman's coefficient) represents a negative correlation
(12.9\% of total Candida) in the Italian study, in which the majority of specimens were collected from patients with neutropenia [2]. Another example is the prevalence of Candida tropicalis in a tertiary care centre in India (46\% of total) [30]. These incongruous findings of NAC distribution among different geographical zones, and even in different facilities in the same zone as in our country, highlight the importance of sample choice, antimicrobial prescription habits, the studied population, the unit(s) involved and the time frame of the study.

\section{Distribution of different Candida species among the departments}

The difference in the isolation of different Candida species among the departments was statistically significant in our hospital $(P=0.005)$ (Table 5). CA was the most common among all departments, with the highest proportion in the OBGYN department (91.4\%), followed by the paediatric department (84.8\%). In the Italian study, CA was found to be the most common Candida species (72.7\%), with the following distribution among the different departments: $64.2 \%$ in haematopoietic stem cell transplant units, $71.1 \%$ in the ICU, and $83.7 \%$ in paediatrics $(P=0.005$ and 0.01 when compared to paediatrics respectively) [2].

With regard to NAC in our series, Candida glabrata prevailed in the IM department (15.7\% of total Candida). Likewise, in the Turkish study, investigators found Candida glabrata as the most common NAC in all departments, mostly in the infectious diseases department (40\% of NAC) [29].

In critical care, the most common speciated NAC after Candida glabrata in our hospital was Candida famata 
(12.4\% of NAC), while in the study by Ece et al. [29], the most common NAC in the critical care/anaesthesiology department was Candida krusei (40\% of NAC).

Candida parapsilosis is known to be a bloodstream isolate, and a common NAC in the paediatric population [31]. Our paediatric department had the highest rate of Candida parapsilosis (25\% of NAC) compared to the other departments, but this was still less common than Candida glabrata (33\% of NAC).

Therefore, the relative distribution of Candida species among different departments of the same hospital differs, with less CA in departments of the severely sick or immunocompromised patients. Among NAC, departments with critically ill and neutropenic patients had more non-glabrata NAC in their fungal ecology.

\section{Correlations between antifungal consumption and Candida species distribution}

The correlation between antifungal consumption and Candida species distribution in the literature is scarce. In a prospective multicentre French surveillance program on yeast bloodstream infections implemented in the ICU, haematology and surgery departments, involving all age groups, Lortholary et al. found that the use of echinocandins decreased CA emergence from 56 to $21 \%$ relatively to NAC [32].

Our study revealed that increasing overall consumption of antifungals and specifically azoles correlated positively with NAC, especially Candida glabrata. The use of azoles may exert a selection pressure, suppressing CA and fostering the growth of NAC since they are active against CA more than NAC [32].

Dagi et al. [33] determined the minimum inhibitory concentration $\left(\mathrm{MIC}_{90}\right)$ of different antifungals against 200 Candida spp. isolates from bloodstream infections between 2010 and 2013 at Selcuk University Hospital in Turkey. The $\mathrm{MIC}_{90}$ of antifungals against Candida glabrata was as such: $4 \mu \mathrm{g} / \mathrm{mL}$ for fluconazole, $0.12 \mu \mathrm{g} / \mathrm{mL}$ caspofungin and $0.06 \mu \mathrm{g} / \mathrm{mL}$ for anidulafungin. Based on this susceptibility of Candida glabrata to echinocandins, one would expect a negative correlation between them. However, our data did not show a negative correlation between the echinocandins and Candida glabrata. This lack of negative correlation may have been due to sampling bias whereby the majority of our specimens were from urine (Fig. 1). Despite moderate distribution of echinocandins into the kidneys, they exhibit negligible concentrations $(<2 \%)$ of intact drug in human urine. Thus, Candida growing in urine might not be affected by the use of echinocandins [34].

Unfortunately, antifungal susceptibility data of different Candida species is lacking in our study. This could have aided in interpreting Spearman's correlations, especially that reports about echinocandin resistance in
Candida glabrata have started to appear [35]. Alexander et al. tested the echinocandin susceptibility of all Candida species causing bloodstream infections between 2001 and 2010 at Duke University Hospital in the US and found that echinocandin resistance increased from 4.9 to $12.3 \%$ [35].

The Spearman's coefficient showed a weaker positive correlation of amphotericin $\mathrm{B}$ with the emergence of NAC compared to azoles $(\mathrm{SC}=0.27$ and 0.38 , respectively) and a negative correlation between amphotericin $\mathrm{B}$ and Candida glabrata ( $\mathrm{SC}=-0.003$ ). This could be explained by the fact that amphotericin B, unlike azoles, is equally active against most Candida species, including CA and NAC (except Candida dubliniensis) or more specifically Candida glabrata [36]. So its use in wards, such as paediatrics and oncology, might have buffered the selection pressure exerted by the azoles in promoting the growth of NAC or Candida glabrata.

The isolation of non-speciated Candida and Candida famata in our series ( $2 \%$ of total isolates, and $9 \%$ of $\mathrm{NAC}$ ) is noteworthy. The emergence of non-classical Candida is increasingly reported in the literature. Between 2012 and 2015, a new species called Candida auris was reported from 3 continents, South East Asia (Pakistan, India), South Africa and South America (Venezuela), and has shown resistance to several classes of antifungals [37]. This change in Candida species epidemiology is in no doubt driven by antifungal consumption and thus highlights the importance of implementing antifungal stewardship.

\section{Limitations and strengths}

One limitation is that this study was single-centred, so our results could not be representative of the whole country. Another was the lack of antifungal susceptibility data in our facility that would have clarified the correlation between antifungal consumption and Candida species distribution. Another issue was that in some wards like the IM ward, some patients might have been clinically unstable necessitating ICU admission, or they might have been transferred early from the critical care unit when still unstable and on broad-spectrum antimicrobials due to shortage in ICU beds. These patients were counted as IM patients not as ICU patients. Consequently, the isolation of specific Candida species and antifungal consumption in the IM ward were affected by this occasional mixing of clinically stable and unstable patients. Nevertheless, this study is among the first studies in the region that describes antifungal consumption and relates it to Candida species distribution on hospital setting. We observed a clear difference in both elements among different wards, yet this difference would have been more significant if mixing in patient populations did not occur. In addition, this study describes overall 
Candida ecology in one facility rather than being limited to Candida-related bloodstream infections.

\section{Conclusion}

Different Candida species are distributed unequally among the hospital departments of our facility, and this correlates with antifungal consumption. Our study highlights the need for benchmarking antifungal use, and standardisation of the metrics. Yet, the relationship between the changing Candida ecology according to the antifungal use highlights the strong need for antifungal stewardship to prevent reaching the era of predominant multi-drug resistant Candida.

\section{Abbreviations \\ ATC: Anatomical Therapeutic Chemical; CA: Candida albicans; DDD: Defined Daily Dose; DOT: Days of Therapy; ICU: Intensive Care Unit; IM: Internal Medicine; MGH: Makassed General Hospital; MIC: Minimum Inhibitory Concentration; NAC: Non-albicans Candida; OBGYN: Obstetrics/Gynaecology; PD: Patient Days; RDD: Recommended Daily Dose; SC: Spearman's Coefficient; WHO: World Health Organisation}

\section{Acknowledgements}

We would like to acknowledge Mr. Ziad Itani for providing technical assistance in data retrieval.

\section{Funding}

None.

\section{Availability of data and materials}

The data that support the findings of this study are available from Makassed General Hospital but restrictions apply to the availability of these data, which were used under license for the current study, and so are not publicly available. Data are however available from the authors upon reasonable request and with permission of Makassed General Hospital.

\section{Authors' contributions}

LA, MS and KZ were responsible for data collection. HT and ND were responsible for data analysis. DA was responsible for result analysis and manuscript editing. $\mathrm{AM}$ and $\mathrm{Al}$ were in charge of study design and result analysis. TJ contributed to the microbiological analysis. RM majorly contributed to the study design and manuscript editing. All the authors have equally contributed to the drafting and reviewing of the manuscript. All authors read and approved the final manuscript.

\section{Ethics approval and consent to participate}

The Makassed General Hospital Institutional Review Board Committee approved this study. No informed consent was required due to the retrospective nature of this study. During the data collection phase, only subject case numbers were included. At a later stage, a different number was assigned to each of our cases to safeguard subject privacy. The contributing authors only performed data entry and analysis as well as the drafting of the manuscript.

\section{Consent for publication}

Not applicable.

\section{Competing interests}

The authors declare that they have no competing interests.

\section{Publisher's Note}

Springer Nature remains neutral with regard to jurisdictional claims in published maps and institutional affiliations.

\section{Author details}

${ }^{1}$ Infectious Diseases and Antimicrobial Stewardship Clinical Pharmacist, Makassed General Hospital, Beirut, Lebanon. ${ }^{2}$ Department of Internal Medicine, American University of Beirut, Beirut, Lebanon. ${ }^{3}$ Pharmacy Department, Makassed General Hospital, Beirut, Lebanon. ${ }^{4}$ Department of
Internal Medicine, Makassed General Hospital, Beirut, Lebanon. ${ }^{5}$ Division of Hematology/Oncology, Department of Internal Medicine, Makassed General Hospital, Beirut, Lebanon. ${ }^{6}$ Department of Laboratory Medicine, Makassed General Hospital, Beirut, Lebanon. ${ }^{7}$ Middle East Institute of Health, Bsalim, Beirut, Lebanon. ${ }^{8}$ Pharmacy Department, Makassed General Hospital, Beirut, Lebanon. ${ }^{9}$ Head of Antimicrobial Stewardship Program, Makassed General Hospital, Beirut, Lebanon.

Received: 1 November 2017 Accepted: 8 November 2018

Published online: 20 November 2018

\section{References}

1. Deorukhkar SC, Saini S. Non albicans Candida species: a review of epidemiology, pathogenicity and antifungal resistance. Pravara Medical Review. 2015;7(3):7-15.

2. Fadda ME, Podda GS, Pisano MB, Deplano M, Cosentino S. Prevalence of Candida species in different hospital wards and their susceptibility to antifungal agents: results of a three year survey. J Prev Med Hyg. 2008:49(2):69-74.

3. Milazzo L, Peri AM, Mazzali C, Grande R, Cazzani C, Ricaboni D, et al. Candidaemia observed at a university hospital in Milan (northern Italy) and review of published studies from 2010 to 2014. Mycopathologia. 2014; 178(3-4):227-41. https://doi.org/10.1007/s11046-014-9786-9.

4. Yang CW, Barkham TM, Chan FY, Wang Y. Prevalence of Candida species, including Candida dubliniensis, in Singapore. J Clin Microbiol. 2003;41(1): 472-4.

5. Ortega M, Marco F, Soriano A, Almela M, Martinez JA, Lopez J, et al. Candida species bloodstream infection: epidemiology and outcome in a single institution from 1991 to 2008. J Hosp Infect. 2011;77(2):157-61. https://doi. org/10.1016/j.jhin.2010.09.026.

6. Chandrasekar P. Management of invasive fungal infections: a role for polyenes. J Antimicrob Chemother. 2011;66(3):457-65. https://doi.org/10.1093/jac/dkq479.

7. Krcmery V, Kalavsky E. Antifungal drug discovery, six new molecules patented after 10 years of feast: why do we need new patented drugs apart from new strategies? Recent Pat Antiinfect Drug Discov. 2007;2(3):182-7.

8. Fournier P, Schwebel C, Maubon D, Vesin A, Lebeau B, Foroni L, et al. Antifungal use influences Candida species distribution and susceptibility in the intensive care unit. J Antimicrob Chemother. 2011;66(12):2880-6. https://doi.org/10.1093/jac/dkr394.

9. Falagas ME, Roussos N, Vardakas KZ. Relative frequency of albicans and the various non-albicans Candida spp among candidemia isolates from inpatients in various parts of the world: a systematic review. Int J Infect Dis. 2010;14(11):e954-66. https://doi.org/10.1016/j.jijid.2010.04.006.

10. Araj GF, Asmar RG, Avedissian AZ. Candida profiles and antifungal resistance evolution over a decade in Lebanon. J Infect Dev Ctries. 2015;9(9):997-1003. https://doi.org/10.3855/jidc.6550.

11. Guinea J. Global trends in the distribution of Candida species causing candidemia. Clin Microbiol Infect. 2014;20(Suppl 6):5-10. https://doi.org/10 1111/1469-0691.12539.

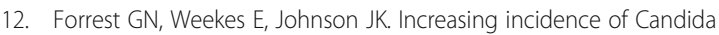
parapsilosis candidemia with caspofungin usage. J Inf Secur. 2008;56(2):1269. https://doi.org/10.1016/j.jinf.2007.10.014.

13. Paugam A, Baixench MT, Taieb F, Champagnac C, Dupouy-Camet J. Emergence of Candida parapsilosis candidemia at Cochin hospital. Characterization of isolates and search for risk factors. Pathol Biol (Paris). 2011;59(1):44-7. https://doi.org/10.1016/j.patbio.2010.08.009.

14. Von Elm E, Altman DG, Egger M, Pocock SJ, Gøtzsche PC, Vandenbroucke $J P$, Strobe Initiative. The Strengthening the Reporting of Observational Studies in Epidemiology (STROBE) statement: guidelines for reporting observational studies. PLoS medicine. 2007;4(10):e296.

15. Agha M, Agha SA, Sharafat S, Barakzai R, Zafar NU, Khanani MR, Mirza MA. API 20C: a reliable and rapid diagnostic tool for fungal infections. Gomal J Med Sci. 2012;10:237-40

16. Pappas PG, Kauffman CA, Andes DR, Clancy CJ, Marr KA, Ostrosky-Zeichner L, Reboli AC, Schuster MG, Vazquez JA, Walsh TJ, Zaoutis TE. Clinical practice guideline for the management of candidiasis: 2016 update by the Infectious Diseases Society of America. Clin Infect Dis. 2015;62(4):e1-50.

17. Freifeld AG, Bow EJ, Sepkowitz KA, Boeckh MJ, Ito Jl, Mullen CA, Raad II, Rolston KV, Young JA, Wingard JR. Clinical practice guideline for the use of antimicrobial agents in neutropenic patients with cancer: 2010 update by the Infectious Diseases Society of America. Clin Infect Dis. 2011;52(4):e56-93. 
18. Lewis RE. Current concepts in antifungal pharmacology. Mayo Clin Proc. 2011;86(8):805-17.

19. Griffith M, Postelnick M, Scheetz M. Antimicrobial stewardship programs: methods of operation and suggested outcomes. Expert Rev Anti-Infect Ther. 2012;10(1):63-73. https://doi.org/10.1586/eri.11.153.

20. WHO Collaborating Centre for Drug Statistics Methodology. Guidelines for ATC classification and DDD assignment, vol. 2012. Oslo: World Health Organization; 2013. p. 250.

21. Ibrahim OM, Polk RE. Antimicrobial use metrics and benchmarking to improve stewardship outcomes: methodology, opportunities, and challenges. Infect Dis Clin N Am. 2014;28(2):195-214. https://doi.org/10. 1016/j.idc.2014.01.006.

22. Guillot J, Lebel D, Roy H, Ovetchkine P, Bussieres JF. Usefulness of defined daily dose and days of therapy in pediatrics and obstetrics-gynecology: a comparative analysis of antifungal drugs (2000-2001, 2005-2006, and 20102011). J Pediatr Pharmacol Ther. 2014;19(3):196-201. https://doi.org/10.5863/ 1551-6776-19.3.196.

23. Valerio M, Munoz P, Rodriguez CG, Caliz B, Padilla B, Fernandez-Cruz A, et al. Antifungal stewardship in a tertiary-care institution: a bedside intervention. Clin Microbiol Infect. 2015;21(5):492 e1-9. https:/doi.org/10.1016/j.cmi.2015.01.013.

24. Singer AC, Shaw $H$, Rhodes $V$, Hart A. Review of antimicrobial resistance in the environment and its relevance to environmental regulators. Front Microbiol. 2016;7:1728. https://doi.org/10.3389/fmicb.2016.01728.

25. Alothman AF, Althaqafi AO, Matar MJ, Moghnieh R, Alenazi TH, Farahat FM, et al. Burden and treatment patterns of invasive fungal infections in hospitalized patients in the Middle East: real-world data from Saudi Arabia and Lebanon. Infect Drug Resist. 2017;10:35-41. https://doi.org/10.2147/IDR.S97413.

26. Dumartin C, Rogues AM, Heriteau F, Pefau M, Bertrand X, Jarno P, et al. Antifungal use in France: first multicentre survey in haematology, intensive care units and at hospital level in 2012.Presented at ESCMID in 2016.

27. Gross BN, Steib-Bauert M, Kern WW, Knoth H, Borde JP, Krebs S, et al. Hospital use of systemic antifungal drugs: a multi-center surveillance update from Germany. Infection. 2015;43(4):423-9. https://doi.org/10.1007/s15010-015-0742-5.

28. European Centre for Disease Prevention and Control. Surveillance of antimicrobial consumption in Europe 2012. Stockholm: ECDC. p. 2014.

29. Ece G. Distribution of yeast-like fungi at a university hospital in Turkey. Jundishapur J Microbiol. 2014;7(12):e13141. https//doi.org/10.5812/jmm.13141.

30. Rajeevan S, Thomas M, Appalaraju B. Characterisation and antifungal susceptibility pattern of Candida species isolated from various clinical samples at a tertiary care Centre in South India. Indian J Microbiol Res. 2016; 3(1):53-7. https://doi.org/10.5958/2394-5478.2016.00014.5.

31. van Asbeck EC, Clemons KV, Stevens DA. Candida parapsilosis: a review of its epidemiology, pathogenesis, clinical aspects, typing and antimicrobial susceptibility. Crit Rev Microbiol. 2009;35(4):283-309. https://doi.org/10.3109/ 10408410903213393.

32. Lortholary O, Desnos-Ollivier M, Sitbon K, Fontanet A, Bretagne S, Dromer F, et al. Recent exposure to caspofungin or fluconazole influences the epidemiology of candidemia: a prospective multicenter study involving 2,441 patients. Antimicrob Agents Chemother. 2011;55(2):532-8. https://doi. org/10.1128/AAC.01128-10

33. Dagi HT, Findik D, Senkeles C, Arslan U. Identification and antifungal susceptibility of Candida species isolated from bloodstream infections in Konya. Turkey Ann Clin Microbiol Antimicrob. 2016;15(1):36. https://doi.org/ 10.1186/s12941-016-0153-1.

34. Stone JA, Xu X, Winchell GA, Deutsch PJ, Pearson PG, Migoya EM, et al. Disposition of caspofungin: role of distribution in determining pharmacokinetics in plasma. Antimicrob Agents Chemother. 2004;48(3):815-23.

35. Alexander $B D$, Johnson $M D$, Pfeiffer $C D$, Jimenez-Ortigosa C, Catania J, Booker R, et al. Increasing echinocandin resistance in Candida glabrata: clinical failure correlates with presence of FKS mutations and elevated minimum inhibitory concentrations. Clin Infect Dis. 2013;56(12):1724-32. https://doi.org/10.1093/cid/cit136.

36. Sabatelli F, Patel R, Mann PA, Mendrick CA, Norris CC, Hare R, et al. In vitro activities of posaconazole, fluconazole, itraconazole, voriconazole, and amphotericin B against a large collection of clinically important molds and yeasts. Antimicrob Agents Chemother. 2006;50(6):2009-15. https://doi.org/ 10.1128/AAC.00163-06.

37. Lockhart SR, Etienne KA, Vallabhaneni S, Faroogi J, Chowdhary A, Govender NP, et al. Simultaneous emergence of multidrug-resistant Candida auris on 3 continents confirmed by whole-genome sequencing and epidemiological analyses. Clin Infect Dis. 2017;64(2):134-40. https://doi.org/10.1093/cid/ciw691.

\section{Ready to submit your research? Choose BMC and benefit from}

- fast, convenient online submission

- thorough peer review by experienced researchers in your field

- rapid publication on acceptance

- support for research data, including large and complex data types

- gold Open Access which fosters wider collaboration and increased citations

- maximum visibility for your research: over $100 \mathrm{M}$ website views per year

At BMC, research is always in progress.

Learn more biomedcentral.com/submissions 